\title{
An algorithm of good clinical practice to reduce intra-hospital 90-day mortality and need for Intensive Care Unit transfer: a new approach for septic patient management
}

\author{
Silvia Spoto, ${ }^{1}$ Sebastiano Costantino, ${ }^{1}$ Marta Fogolari, ${ }^{2}$ Emanuele Valeriani, ${ }^{3}$ Massimo Ciccozzi, ${ }^{4}$ Silvia Angeletti ${ }^{2}$ \\ ${ }^{1}$ Internal Medicine Department, University Campus Bio-Medico of Rome; ${ }^{2}$ Unit of Clinical Laboratory Science, University \\ Campus Bio-Medico of Rome; ${ }^{3}$ Internal Medicine Department, University G. D’Annunzio, Chieti; ${ }^{4}$ Unit of Medical Statistics \\ and Molecular Epidemiology, University Campus Bio-Medico of Rome, Italy
}

\begin{abstract}
Sepsis accounts for $50 \%$ of intra-hospital mortality. Septic shock is diagnosed in $2 \%$ of patients with half of these needing for Intensive Care Unit (ICU) transfer. An algorithm was applied for mortality, need for intensive care transfer and length of stay decrease. The observational study was performed on 231 consecutive enrolled patients with sepsis or septic shock admitted to the University Campus Bio-Medico of Rome. The algorithm was based on good clinical practice application of antimicrobial stewardship. Data were compared with data from comparable population of National and European surveys. In the study group, the global mortality rate was $26.8 \%$ (3.9\% was intra-hospital and 23\% 90-d mortality), need of ICU transfer was registered in $21.6 \%$, and the median length of stay was 15 days. Globally, intra-hospital and 90-day mortality, were significantly lower than at national level $(26.8 \%$ vs $63.6 \%, \mathrm{P}<0.0001 ; 3.9 \%$ vs $25 \%, \mathrm{P}<0.0001$ and $23 \%$ vs $37.5 \%, \mathrm{P}=0.0092)$. Need for ICU transfer in patients with septic shock was significantly lower than in the English survey $(31.9 \%$ by $v s 80.8 \% \mathrm{P}<0.0001)$.

A significant decrease of intra-hospital, 90-d mortality and need of ICU transfer was achieved.
\end{abstract}

\section{Introduction}

Sepsis is a condition, affecting 19 million of people worldwide per year even if the real incidence, presum-

Correspondence: Silvia Spoto, Internal Medicine Department, University Campus Bio-Medico of Rome, via Alvaro del Portillo 200, Rome, Italy.

Tel.: +39.06.225411461. E-mail: s.spoto@unicampus.it

Key words: Sepsis; mortality; Intensive Care Unit transfer; algorithm; antimicrobial stewardship.

Acknowledgments: the authors thank Dr. Ranieri Guerra, MD, for the scientific support and the critical revision of the manuscript.

Contributions: SS, SC, SA, EV, MC, manuscript writing and revising; SS, SC, EV, analysis of clinical data and patient management; SA, MF, laboratory test. All authors contributed to data analysis, drafting and clinically revision of the manuscript and agreed to be responsible for any aspect of the manuscript. All authors read and approved the final manuscript.

Conflict of interests: the authors declare no potential conflict of interests.

Received for publication: 25 September 2019.

Accepted for publication: 7 October 2019.

This work is licensed under a Creative Commons Attribution NonCommercial 4.0 License (CC BY-NC 4.0).

${ }^{\circ}$ Copyright: the Author(s), 2020

Licensee PAGEPress, Italy

Italian Journal of Medicine 2020; 14:14-21

doi:10.4081/itjm.2020.1215 ably far higher, is projected to increase by $1.5 \%$ per year. ${ }^{1,2}$ Sepsis arises when the response to an infection injures patient's tissue and organs and represents the $10^{\text {th }}$ leading cause of death in the United States.

About $28.6 \%$ of patients with sepsis die, reaching $40-60 \%$ in case of septic shock, even when optimal treatment is given ${ }^{3,4}$ accounting for $50 \%$ of intra-hospital mortality ${ }^{5,6}$ with average costs over 24 billion

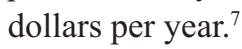

Septic shock is diagnosed in $2 \%$ of patients admitted to the hospital with half of these needing treatment in Intensive Care Unit (ICU), representing 10\% of all ICU admissions. ${ }^{2}$

Mortality and costs of patients with sepsis depend on several factors: appropriate and prompt management, invasiveness of microbial pathogens, source of infection and presence of risk factors. Inadequate antimicrobial therapy and antimicrobial resistance are extremely linked, increasing the rate of severe infections such as in case of ESKAPE pathogens, for their ability to escape the antimicrobial action of antibiotics that represents a new challenge for transmission and resistance spread (Enterococcus faecium, Staphylococcus aureus, Klebsiella pneumoniae, Acinetobacter baumannii, Pseudomonas aeruginosa and Enterobacter spp.) which impact on mortality and global economy. ${ }^{8,9}$

The risk is higher in people with weakened immune systems, chronic illnesses [diabetes, AIDS, cancer, chronic obstructive pulmonary disease (COPD) and kidney or liver disease], elderly people, infants and children, severe burn or physical trauma. ${ }^{10}$ 
Antimicrobial resistance (AMR) is highly influenced by antibiotic consumption with a rapid increase in the use of last generation compounds as glycylcyclines, oxazolidinones, carbapenems, and polymyxins.

The Infectious Disease Society of America (IDSA) defines the antimicrobial stewardship (AMS) as a set of interventions to decrease the inappropriate use of antimicrobials by encouraging the correct choice in terms of antimicrobial class, dose, duration of therapy, and way of administration. On this basis, a prompt and accurate clinical and microbiological diagnosis is necessary to start appropriate therapy as soon as possible. Obtaining a good medical history represents the first step to make an accurate diagnosis, allowing to reach diagnosis in $76-90 \%$ of cases. The following adequate physical examination, laboratory or imaging tests allow the increase of the percentage of diagnosis. ${ }^{11}$ The choice of the appropriate antimicrobial therapy requires the identification of the site of infection and of the potential causal pathogens. Collection of biological samples (specimens from suspected site of infection and three sets of blood culture) has to be performed within 1 hour from symptoms onset before starting antimicrobial therapy. ${ }^{12,13}$ Simultaneously, some bioumoral markers, such as procalcitonin (PCT) have been proposed to improve sensitivity and specificity of the diagnosis and guide the empirical approach. ${ }^{14}$

PCT monitoring has been demonstrated to be useful for antimicrobial therapy starting and discontinuation. It has been estimated that PCT monitoring leads to cost savings of about $9 \%$ per patient per hospitalization, due to decreased antibiotic therapy duration, hospital and ICU lengths of stay and global reduction in pharmacy costs. ${ }^{15}$ In a recent study involving 14,000 ICU patients in 75 countries, Gram-negative bacteria were isolated in $62 \%$ of patients, Gram-positive bacteria in $47 \%$, and fungi in $19 \%$. S. aureus and Streptococcus pneumoniae are the most common Gram-positive isolates, whereas Escherichia coli, Klebsiella species, and P. aeruginosa predominate among Gram-negative isolates. ${ }^{16}$ Blood cultures are typically positive in only one third of cases, and in up to a third of cases, cultures from all sites are negative. Especially in case of negative culture, PCT measurement can guide bacterial diagnosis, differentiating Gram-negative, Gram-positive and fungal infections based on PCT cut-off values, to set up a more oriented empirical therapy. ${ }^{17}$

Patients hospitalized for sepsis had an average length of stay of $75 \%$ longer than patients hospitalized for other conditions, with a median length of stay (LOS) of 22 days. After sepsis recovery, a severe disability could be residual; $50 \%$ of the patients are likely to be discharged home, the remaining could need transfer to short- or long-term care facility. ${ }^{18,19}$ Multiple studies have shown that patients managed by a hospitalist have a shorter length of stay and lower hospital costs than those managed by other physicians. ${ }^{20}$

In 2009, Peterson et al. reviewed 18 reports that compared care by hospitalists $v s$ non hospitalists; in 15 reports, there were no differences in the 30-day death rate or the readmission rate; in 3 reports, mortality and readmissions were less frequent for hospitalist patients; this variability could be influenced by hospitalist skills..$^{20}$ Cost-effective care by hospitalists does not diminish the quality of care as measured by in-hospital mortality or the readmission rate. ${ }^{20}$

For septic patient management we elaborated a clinical methodology based on the application of a new clinical and diagnostic algorithm and the institution of an AMS program with the aim to reduce mortality, need for intensive care transfer and length of stay, as indicators of antimicrobial stewardship efficiency.

\section{Materials and Methods}

The observational study was performed on 231 randomly consecutive enrolled patients with diagnosis of sepsis or septic shock admitted to the Department of Internal Medicine with sub-intensive care unit and General Surgery of the University Hospital Campus Bio-Medico of Rome, between 2016 and 2018. Informed consent was obtained from all patients prior enrollment to the study. The study was approved by the Ethical Committee of the University Hospital Campus Bio-Medico of Rome. Inclusion criteria were as follows: patients with sepsis and septic shock defined by Third Consensus Sepsis Conference. ${ }^{21}$ Exclusion criteria were the absence of informed consent and pregnancy. Clinical evaluation was always performed by the same medical staff including hospitalists represented by Internists practicing Hospital Medicine with specialized training in infectious diseases.

At inclusion, the day, demographic characteristics, prior or current use of antibiotics, risk factors for sepsis, clinical presentation, and bioumoral parameters were recorded. The sequential organ failure assessment (SOFA) score and the quick SOFA (qSOFA) score were calculated at inclusion. ${ }^{21}$ Furthermore, data on microbiological culture/isolation and resistant phenotype, were collected.

The primary outcome was to reduce mortality, need for intensive care transfer and length of stay, as indicators of antimicrobial stewardship efficiency.

Mortality rates from our study were compared with data from a multi-centric national study performed on septic patients in Internal Medicine Departments by Mirijello et al. ${ }^{22}$

Data on need of ICU transfer in the study group were compared with European data from an English survey performed on septic shock patients by Mouncey et al..$^{23}$ 
The clinical methodologies used for algorithm building are reported in Table 1. ${ }^{24-32}$

Following the proposed algorithm, showed in Figure 1, the patient with suspected acute infection was evaluated by hospitalists belonging to the AMS clini- cal staff who is in charge of suggesting laboratory biomarkers order (especially PCT), microbiological cultures and pharmacokinetic (PK) monitoring, to improve diagnosis and optimize antimicrobial therapy. Daily clinical revaluation and PCT measurement dur-

Table 1. Clinical methodology used for algorithm building.

a Accurate diagnosis and set up of an appropriate and adequate antimicrobial therapy

b The choice of the appropriate antimicrobial therapy requires the identification of the site of infection and of the potential causal pathogens, risk factors of the patient, colonization status for ESKAPE pathogens and PCT value

c Identification of the colonization status for ESKAPE pathogens before the development of clinical infection, to direct the treatment in case of prophylaxis or clinical infection

d Discrimination between real infection and colonization by clinical presentation, positivity of bioumoral markers and/or cultures

$\mathrm{e}^{24}$ Screening swabs are reserved for patients at high risk of ESKAPE pathogens colonization on Hospital Admission: residence in a longterm-care facility, hospitalization - within 1 month -, recent antibiotic exposure - with 3 months -, age $>70$ years, history of cerebrovascular accidents, according to Tseng et al. (2017) or even coming from other hospitals or Emergency room or critical illness in our University Hospital

f Hit hard and early in case of clinical infection presenting in colonized patients by appropriate and early administration of antimicrobial therapy

$\mathrm{g}^{25}$ Avoid bacterial cultures of samples collected from drainage catheters in place for more than $48 \mathrm{~h}$ yielding potentially misleading results and inappropriate overtreatment

$\mathrm{h}^{24}$ Avoid Clostridioides difficile infection, limiting the prescription of clindamycin, macrolides, fluoroquinolones and II-III generation cephalosporins, carbapenems, proton pump inhibitors; stopping cephazolin prophylaxis at the end of the surgery and improving nutrition

$i^{26}$ Prefer bedside evaluation to telephone consultation

$\mathrm{j}^{27}$ Consider the ability of the antimicrobial compounds to arrive at the infectious site by evaluation of the albumin level, the body mass index, the presence of renal failure, lipophilic and hydrophilic characteristics

$\mathrm{k}^{28}$ Calculate the daily dose evaluating the estimated glomerular filtration rate in case of renal failure

1 As soon as the causal pathogen is isolated, switch from empirical to target therapy evaluating if a de-escalation is appropriate; switch from intravenous to oral administration within $48-72 \mathrm{~h}$ if possible; shorten the duration of therapy as little as possible

$\mathrm{m}$ Provide combination therapy in severe, or suspected ESKAPE infections, always in course of colistin and rifampicin, and the use of broadspectrum antibiotics, also in case of monomicrobic culture

$\mathrm{n}$ Stop therapy after short course of antimicrobial therapy (5 days) at the regression of clinical signs of local or systemic infection, in the absence of purulent collection at imaging (source control), at PCT decrease by $80 \%$ or more of its peak value, or to $<0.5 \mathrm{ug} / \mathrm{L}$, or in case of pneumonia to value lower than $0.1 \mathrm{ug} / \mathrm{L}^{29}$

o Continue therapy for 7-10 days in critically ill, immunocompromised patients and in case of MDR infection, with poor prognosis predictors, following PCT value decrease $\mathrm{e}^{30}$

p Dose PCT daily for the first five days from antimicrobial therapy start as guide for cessation, since short-course demonstrated to be as effective as the long-course one 29,30 $^{29}$

q Provide longer courses of antimicrobial therapy ( $>5$ days) in case of infections by Staphylococcus aureus, Pseudomonas aeruginosa, Legionella spp and Pneumocystis spp (21 days), C. difficile (10 days or prolong by further 10 days after stopping of antimicrobial therapy for other causes) and systemic fungal infections (14 days from last negative blood culture). Longer courses are also recommended in case of endocarditis or suppurative thrombophlebitis (4-6 weeks), osteomyelitis (6 weeks-3 months), Streptococcus pneumoniae meningitis (2 weeks), Lysteria spp meningitis ( $\geq 3$ weeks), Gram-negative bacilli meningitis (3 weeks), or uncomplicated catheter related bloodstream infections by Enterococcus spp and Gram-negative bacilli (1-2 weeks)

r Tailor antifungal therapy for each patient. Antifungal therapy is recommended in septic shock or febrile neutropenia. Consider empirical therapy in case of febrile patients with fungal colonization or C. difficile infection and low PCT value

$\mathrm{s}^{31}$ Get bronchoalveolar lavage for molecular detection of respiratory viruses, if clinically indicated, especially in ICU setting, for etiology definition and limit antibiotic prescribing

$\mathrm{t} \quad$ For optimal therapy PK monitoring is suggested daily or every other day for at least aminoglycosides, vancomycin and linezolid (especially in neoplastic patients), to avoid the onset of adverse effects, as acute kidney injury or myelotoxicity, the delay of patient's discharge and to reduce costs

u Source control (involving imaging studies, surgical or percutaneous drainage of body collections, removing infected devices, where indicated) is mandatory

PCT, procalcitonin; MDR, multi-drug resistant; ICU, Intensive Care Unit; PK, pharmacokinetic. 
ing the first five days were used to follow antimicrobial therapy. ${ }^{32}$

On the fifth day, the regression of clinical signs of local or systemic infection, the absence of critical illness, immunocompromised status, resistant-pathogen infection, poor prognosis predictors, the PCT decrease by $80 \%$ or more of its peak value, or to $<0.5 \mathrm{ug} / \mathrm{L}$, or in case of pneumonia to value lower than $0.1 \mathrm{ug} / \mathrm{L}$, allowed to stop the therapy. ${ }^{19,29,32}$

Antimicrobial therapy (AMT) for 7-10 days is suggested in case of critically ill, immunocompromised patients or with poor prognosis predictors and in case of multi-drug resistant (MDR)-ESKAPE infection, even in case of PCT value decrease (Figure 1). ${ }^{29,33-35}$

Longer AMT course is recommended in case of infections by $S$. aureus, $P$. aeruginosa, Legionella spp,
Pneumocystis spp, Clostridioides difficile; systemic fungal infections; in case of endocarditis or suppurative thrombophlebitis; osteomyelitis; $S$. pneumoniae meningitis; Lysteria spp meningitis; Gram-negative bacilli meningitis; uncomplicated catheter-related bloodstream infections by Enterococcus spp and Gram-negative bacilli (Figure 1).

A tailored therapy is suggested in case of positivity for MDR microorganisms at the surveillance swabs, and for specific clinical data such as albumin level, body-mass index, daily estimated glomerular filtration rate (Figure 1).

Adjustment or optimization of the empiric therapy was provided at results cultures receiving. Imaging exams were requested based on the evaluation of specific clinical conditions.

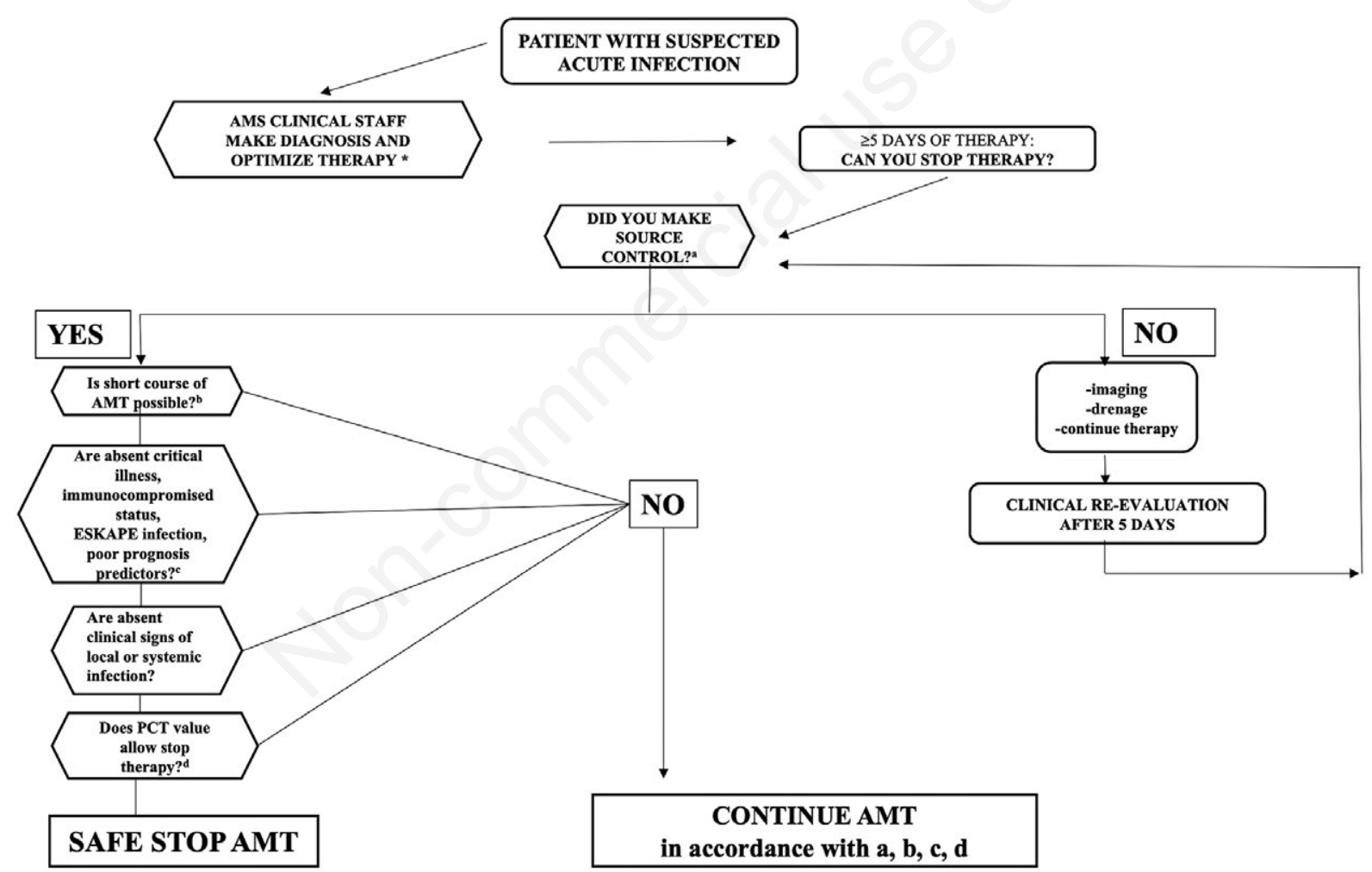

Figure 1. Algorithm for septic patient management. a- Source control by imaging, surgical or percutaneous drainage of body collections, removing infected devices; b-, c- Need for antimicrobial therapy (AMT) courses $>5$ days in case of critically ill, immunocompromised patients or poor prognosis predictors and ESKAPE pathogens infection. In case of Staphylococcus aureus, Pseudomonas aeruginosa, Legionella and Pneumocystis spp prolong by 21 days; Clostridioides difficile infection by 10 days or further 10 days from antimicrobial therapy stop; systemic fungal infections by 14 days from last negative blood culture; endocarditis or suppurative thrombophlebitis by 4-6 weeks; osteomyelitis by 6 weeks-3 months; Streptococcus pneumoniae meningitis by 2 weeks; Lysteria spp meningitis for $\geq 3$ weeks; Gram-negative bacilli meningitis by 3 weeks; uncomplicated catheter-related bloodstream infections by Enterococcus spp and Gram-negative bacilli by 10-14 days; d- Procalcitonin (PCT) decrease by $80 \%$ or more of its peak value, or to $<0.5 \mathrm{ug} / \mathrm{L}$, or in case of pneumonia to value lower than $0.1 \mathrm{ug} / \mathrm{L}$, allows stop therapy at the regression of clinical signs of infection and after source control. *by clinical methodology reported in Table 1. AMS, antimicrobial stewardship; BMI, body mass index; PCT, procalcitonin; eGFR, estimated glomerular filtration rate; PK, antimicrobial pharmacokinetic. 
After algorithm application, clinical indicators as mortality rate, need of ICU transfer, LOS were evaluated. Mortality rate was estimated as in-hospital mortality and 90-day mortality analyzing electronic data as well by phone recall. Need of ICU transfer has been considered during the period from sepsis onset to discharge or death. Patients who died were excluded from the analysis examining LOS. Clinical indicators were compared with those observed in the control population.

For age and LOS median values were evaluated. Mortality and ICU transfer rates were compared using $\chi^{2}$ test for proportions. $P$ values $<0.05$ were considered as statistically significant. Data have been analyzed using Med-Calc 11.6.1.0 statistical package (MedCalc Software, Mariakerke, Belgium).

\section{Results}

The characteristics of the study population are reported in Table 2. Median age was 71 years (64-80), and $120(52 \%)$ were male.

One hundred and one patients $(43.7 \%)$ received chemo-radiotherapy treatment, 21 patients $(11 \%)$ were affected by autoimmune disease or were receiving immunosuppressive treatments (Table 2).

Regarding comorbidities distribution, 60 patients (25.9\%) had chronic kidney failure, 16 patients $(6.9 \%)$ chronic liver failure, 47 patients $(20.3 \%)$ were affected by diabetes, while 46 patients $(19,9 \%)$ by COPD.

Age, sex and comorbidities distribution of study population were compared with those from the multicentric national survey performed by Mirijello et $\mathrm{al}^{22}$ No significant difference was found between the two populations.

Resistant pathogens were isolated in 31 patients (14.7\%) of which MDR-ESKAPE pathogens were detected in 8 patients $(3.4 \%)$, while methicillinresistant $S$. aureus (MRSA) in 4 patients (1.7\%). 94/231 patients $(40.7 \%$ ) had septic shock, while 137 patients (59.3\%) suffered from sepsis (Table 2).

Regarding clinical indicators of interest, need of ICU transfer was registered in 50/231 patients $(21.6 \%)$ while median LOS was 15 days [interquartile range (IQR) 11-26 days] (Table 2).

The global mortality rate was $26.8 \%(62 / 231$ patients) of which 3.9\% (9/231) was intra-hospital and $23 \%$ (53/231) was 90-day mortality (Table 3$)$. In septic shock patients, intra-hospital mortality was $8.5 \%$ (8/94 patients) and 90-day mortality was 38.3\% (36/94 patients), whereas in sepsis intra-hospital was $0.7 \%$ (1/137) and 90-day mortality was $12.4 \%(17 / 137$ patients) (Table 3 ).

In the study group, data of global mortality, intrahospital and 90-day mortality were significantly lower than those reported from the reference Italian survey (26.8\% vs 63.6\%, $\mathrm{P}<0.0001 ; 3.9 \%$ vs $25 \%, \mathrm{P}<0.0001$; and $23 \%$ vs $37.5 \%, \mathrm{P}=0.0092$ ). The survey was per-

Table 2. Demographic and clinical characteristics of study population.

\begin{tabular}{|c|c|}
\hline Variables & Study group $=\mathbf{2 3 1}$ \\
\hline Median age (IQR) & $71(64-80)$ \\
\hline Sex male n. $(\%)$ & $120(52)$ \\
\hline Chemo-radiotherapy in active malignancy n. (\%) & $101(43.7)$ \\
\hline Autoimmune disease/ immunosuppressive therapy n. (\%) & $21(11.0)$ \\
\hline Chronic kidney failure n. (\%) & $60(25.9)$ \\
\hline Chronic liver failure n. (\%) & $16(6.9)$ \\
\hline Diabetes mellitus n. (\%) & $47(20.3)$ \\
\hline COPD n. (\%) & $46(19.9)$ \\
\hline $\begin{array}{l}\text { Resistant pathogens isolation n. (\%) } \\
\text { MDR-ESKAPE pathogen isolation n. (\%) } \\
\text { MRSA isolation n. }(\%)\end{array}$ & $\begin{array}{l}31(14.7) \\
8(3.4) \\
4(1.7)\end{array}$ \\
\hline Sepsis n. $(\%)$ & $137(59.3)$ \\
\hline Septic shock n. (\%) & $94(40.7)$ \\
\hline Need of ICU transfer n. (\%) & $50(21.6)$ \\
\hline $\begin{array}{l}\text { Median LOS in day (IQR) } \\
\text { Median LOS in SS } \\
\text { Median LOS in S }\end{array}$ & $\begin{array}{l}15.5(11-26) \\
14.5(10-25) \\
16.5(11-28)\end{array}$ \\
\hline
\end{tabular}

IQR, interquartile range; COPD, chronic obstructive pulmonary disease; MDR, multi-drug resistant; MRSA, methicillin-resistant Staphylococcus aureus; ICU, Intensive Care Unit; LOS, length of stay; S, sepsis; SS, septic shock. 
formed on a septic patient population comparable for age, sex, comorbidities, hospital and country settings. ${ }^{22}$

Stratifying patients by sepsis and septic shock, in sepsis intra-hospital mortality was $0.7 \%$ vs $26.1 \%$ $(\mathrm{P}<0.0001)$ and 90 -day mortality was $12.4 \%$ vs $45.6 \%$ $(\mathrm{P}<0.0001)$, whereas in septic shock intra-hospital mortality was $8.5 \%$ vs $42.3 \%(\mathrm{P}<0.0001)$ and 90 -day mortality was $38.3 \%$ vs $46.1 \%(\mathrm{P}=0.47)$.

In the study group, the need for ICU transfer was registered in $30 / 94 \%(31.9 \%)$ of patients with septic shock. It was significantly lower than the rate reported in the English survey on 1261 septic shock patients ( $31.9 \%$ vs $80.8 \% \mathrm{P}<0.0001)$.

The median LOS of the study group was 15 days (IQR 11-26 days), in particular it was 14.5 days (IQR 10-25 days) in septic shock patients and 16.5 days (IQR 11-28 days) in sepsis.

\section{Discussion}

Adequate clinical management including prompt diagnosis and tailored therapy represent key factors reducing morbidity and mortality of septic patients. ${ }^{32,36,37}$ In this study, the application of a new algorithm for the clinical management of septic patients determined lower mortality rates in comparison with literature data. ${ }^{19,23,38}$ In particular, the mortality rates were significantly lower than those reported in the multi-centric national survey performed by Mirijello et al. on a comparable population of septic patients for age, sex, comorbidities, hospital and country settings. ${ }^{22}$

Both intra-hospital mortality and 90-day mortality were lower despite the higher complexity of patients of the study group, and it is well known that patients hospitalized for sepsis were then eight times as likely to die during their hospitalization. ${ }^{18}$

The evidence of significant lower intra-hospital mortality in septic patients from the study group, 3.9\% vs $25 \%$, exactly $0.7 \%$ vs $26.1 \%$ in sepsis and $8.5 \%$ vs $42.3 \%$ in septic shock patients, suggests an improvement of clinical management and consequent outcome in these patients. Intra-hospital mortality is a more appropriate indicator to evaluate recovery from sepsis event during the acute phase than 90-day mortality where anti-inflammatory response syndrome or persistent inflammation, immunosuppression and catabolism syndrome can determine poor prognosis. ${ }^{30}$

Regarding 90-days mortality, it resulted significantly lower comparing data with the national survey $(23 \%$ vs $37.5 \%)$ but also with the current literature ranging from $28 \%$ to $50 \%$ with higher percentage in septic shock patients. . $^{3-6}$

In Holland the 28-day mortality, for a septic event, ranged from 19.6 to $25 \% .{ }^{19}$ In England the 28-day mortality, for a septic event, was $25 \%$, while the 90 day mortality was $29.5 \% .{ }^{23}$ In South East Asia the 28-day mortality was $34.61 \%$ in case of patients with severe sepsis and $59.09 \%$ in those with septic shock. ${ }^{38}$

Concerning the need for ICU transfer, the registered percentage was significantly lower than the rate reported in the English survey on septic shock patients $(31.9 \%$ vs $80.8 \% \mathrm{P}<0.0001)$, suggesting that the application of the proposed algorithm could have a significant impact on ICU transfer need. Transfer rate, representing an index of disease severity, has been related to prolonged LOS, risk for AMR pathogen selection or acquisition and health costs increase. $^{18}$

The median LOS of the study group was 15.5 days, comparable to that reported in Holland, England and South East Asia, ranging from 12.5 to 22 days respectively and it was independent from the best clinical management applied but influenced by the complex physiopathology of sepsis. . $^{1923,38}$

Overall, these data suggest that the application of the algorithm allows a tailored clinical management of septic patients.

In the algorithm, some important actions have

Table 3. Global mortality rate, intra-hospital and 90-day mortality rates registered in the study population and after stratification for sepsis and septic shock in comparison with data from a national survey.

\begin{tabular}{|c|c|c|c|}
\hline & $\begin{array}{c}\text { Study population } \\
\mathrm{n}=231 ; S=137 ; S S=94\end{array}$ & $\begin{array}{c}\text { Mirijello } \text { et }_{\text {al. }}{ }^{29} \\
\mathrm{n}=\mathbf{8 8} ; \mathrm{S}=46 ; \mathrm{S} S=26\end{array}$ & $\mathbf{P}^{*}$ \\
\hline Global mortality n (\%) & $62(26.8)$ & $56(63.6)$ & $<0.0001$ \\
\hline Intra-hospital mortality n (\%) & $9(3.9)$ & $22(25)$ & $<0.0001$ \\
\hline Mortality at 90 days n (\%) & $53(23)$ & $33(37.5)$ & 0.0092 \\
\hline Intra-hospital mortality in S n (\%) & $1(0.7)$ & $12(26.1)$ & $<0.0001$ \\
\hline Intra-hospital mortality in SS n (\%) & $8(8.5)$ & $11(42.3)$ & $<0.0001$ \\
\hline Mortality at 90 days in S n (\%) & $17(12.4)$ & $21(45.6)$ & $<0.0001$ \\
\hline Mortality at 90 days in SS n (\%) & $36(38.3)$ & $12(46.1)$ & 0.47 \\
\hline
\end{tabular}

${ }^{*} \chi^{2}$ for proportion. S, sepsis; SS, septic shock. 
been described such as tailored empirical therapy based on the origin of infection and/or accurate etiological diagnosis; respect of the golden hour (prompt request of microbiological cultures and biomarkers and empirical therapy administration); constant clinical management of patients and bioumoral monitoring; empiric therapy switching to pathogen-based therapy; antibiotic therapy duration on the basis of the presence of critical illness, immunocompromised status, resistant pathogens, poor prognosis predictors, and on PCT value monitoring. ${ }^{19,29,32}$

All these represent key factors leading to significant reduction of mortality and need for ICU transfer with a significant positive impact on patient management as well as on hospital cost savings.

This study suggests that the algorithm should be applied in medical and surgery units by a staff of hospitalists dedicated to the clinical management of critically ill septic patients. This appeared to be essential to assure the correct adherence to the algorithm actions, thus increasing the appropriateness of ICU transfer of septic shock patients and decreasing mortality.

\section{Conclusions}

The proposed protocol and algorithm, throughout an accurate diagnosis and a prompt treatment, improved the clinical management of septic patients, as showed by the significant decrease of intra-hospital, 90-day mortality and need of ICU transfer. This was achieved thanks to a strict collaboration among physicians and hospitalists (providing adequate source control and general management of sepsis), laboratory staff (by rapid bioumoral, microbiological cultural reports and PK monitoring), and pharmacists (guaranteeing prompt availability of antimicrobial compounds), thus allowing a tailored antimicrobial therapy administration and an optimal patient management.

It should be desirable that the hospital governance could support these actions by promoting internal antimicrobial stewardship guidelines, monitoring antimicrobial costs, as well as the isolation of antimicrobial resistant microorganisms, LOS and mortality rate for infectious diseases.

\section{References}

1. Adhikari NK, Fowler RA, Bhagwanjee S, Rubenfeld GD. Critical care and the global burden of critical illness in adults. Lancet 2010;376:1339-46.

2. Angus DC, Linde-Zwirble WT, Lidicker J, et al. Epidemiology of severe sepsis in the United States: analysis of incidence, outcome, and associated costs of care. Crit Care Med 2001;29:1303-10.

3. Levy MM, Fink MP, Marshall JC, et al. 2001
SCCM/ESICM/ACCP/ATS/SIS International Sepsis Definitions Conference. Crit Care Med 2003;31:1250-6.

4. National Center for Health Statistics. Data Brief No. 62; 2011. Available from: https://www.cdc.gov/nchs/data/ databriefs/db62.pdf

5. ProCESS Investigators, Yealy DM, Kellum JA, et al. A randomized trial of protocol-based care for early septic shock. N Engl J Med 2014;370:1683-93.

6. Liu V, Escobar GJ, Greene JD, et al. Hospital deaths in patients with sepsis from 2 independent cohorts. JAMA 2014;312:90-2.

7. Scott RD, Sinkowitz-Cochran R, Wise ME, et al. CDC central-line bloodstream infection prevention efforts produced net benefits of at least $\$ 640$ Million during 1990-2008. Health Aff (Millwood) 2014;33:1040-7.

8. Cella E, Ciccozzi M, Lo Presti A, et al. Multi-drug resistant Klebsiella pneumoniae strains circulating in hospital setting: whole-genome sequencing and Bayesian phylogenetic analysis for outbreak investigations. Sci Rep 2017;7:3534.

9. Angeletti S, Cella E, Prosperi M, et al. Multi-drug resistant Pseudomonas aeruginosa nosocomial strains: Molecular epidemiology and evolution. Microb Pathog 2018;123:233-41.

10. Angus DC, van der Poll T. Severe sepsis and septic shock. N Engl J Med 2013;369:840-51.

11. Nash DB. Bedside diagnosis: a new annotated bibliography. Ann Intern Med 1988;108:299-300.

12. Kumar A, Roberts D, Wood KE, et al. Duration of hypotension before initiation of effective antimicrobial therapy is the critical determinant of survival in human septic shock. Crit Care Med 2006;34:6.

13. Ihle BU. Clinical practice guidelines for improving outcomes in sepsis. Heart Lung Circulation 2008;17:S26-31.

14. Spoto S, Cella E, de Cesaris M, et al. Procalcitonin and MR-proadrenomedullin combination with SOFA and qSOFA scores for sepsis diagnosis and prognosis: a diagnostic algorithm. Shock 2018;50:44-52.

15. Kip MM, Kusters R, Ijzerman MJ, Steuten LM. A PCT algorithm for discontinuation of antibiotic therapy is a cost-effective way to reduce antibiotic exposure in adult intensive care patients with sepsis. J Med Econ 2015;18:944-53.

16. Vincent JL, Rello J, Marshall J, et al. International study of the prevalence and outcomes of infection in intensive care units. JAMA 2009;302:2323-9.

17. Sager R, Kutz A, Mueller B, Schuetz P. Procalcitoninguided diagnosis and antibiotic stewardship revisited. BMC Med 2017;15:15.

18. Hall MJ, Williams SN, DeFrances CJ, Golosinskiy A. Inpatient care for septicemia or sepsis: a challenge for patients and hospitals. NCHS Data Brief 2011;62:1-8.

19. de Jong E, van Oers JA, Beishuizen A, et al. Efficacy and safety of procalcitonin guidance in reducing the duration of antibiotic treatment in critically ill patients: a randomised, controlled, open-label trial. Lancet Infect Dis 2016;16:819-27.

20. Peterson MC. A systematic review of outcomes and quality measures in adult patients cared for by hospitalists and nonhospitalists. Mayo Clin Proc 2009;84:248-54.

21. Singer M, Deutschman CS, Seymour CW, et al. The Third International Consensus Definitions for Sepsis and Septic Shock (Sepsis-3). JAMA 2016;315:801-10. 
22. Mirijello A, Tosoni A, Zaccone V, et al. MEDS score and vitamin $\mathrm{D}$ status are independent predictors of mortality in a cohort of Internal Medicine patients with microbiological identified sepsis. Eur Rev Med Pharmacol Sci 2019;23:4033-43.

23. Mouncey PR, Osborn TM, Power GS, et al. Trial of early, goal-directed resuscitation for septic shock. N Engl J Med 2015;372:1301-11.

24. Tseng WP, Chen YC, Yang BJ, et al. Predicting multidrug-resistant Gram-negative bacterial colonization and associated infection on hospital admission. Infect Control Hosp Epidemiol 2017;38:1216-25.

25. Everts RJ, Heneghan JP, Adholla PO, Reller LB. Validity of cultures of fluid collected through drainage catheters versus those obtained by direct aspiration. J Clin Microbiol 2001;39:66-8.

26. Forsblom E, Ruotsalainen E, Ollgren J, Järvinen A. Telephone consultation cannot replace bedside infectious disease consultation in the management of Staphylococcus aureus bacteremia. Clin Infect Dis 2013;56:527-35.

27. Meng L, Mui E, Holubar MK, Deresinski SC. Comprehensive guidance for antibiotic dosing in obese adults. Pharmacotherapy 2017;37:1415-31.

28. Gilbert DN, Eliopoulos GM, Chambers HF, et al. The Sanford guide to antimicrobial therapy. Sperryville, VA, USA: Antimicrobial Therapy, Inc., 2018.

29. Shehabi Y, Sterba M, Garrett PM, et al. Procalcitonin algorithm in critically ill adults with undifferentiated infection or suspected sepsis. A randomized controlled trial. Am J Respir Crit Care Med 2014;190:1102-10.

30. Mathias B, Szpila BE, Moore FA, et al. A review of GM-
CSF therapy in sepsis. Medicine (Baltimore) 2015;94:e2044.

31. Legoff J, Guérot E, Ndjoyi-Mbiguino A, et al. High prevalence of respiratory viral infections in patients hospitalized in an intensive care unit for acute respiratory infections as detected by nucleic acid-based assays. J Clin Microbiol 2005;43:455-7.

32. Spoto S, Valeriani E, Caputo D, et al. The role of procalcitonin in the diagnosis of bacterial infection after major abdominal surgery. Advantage from daily measurement. Medicine (Baltimore) 2018;97:3-8.

33. Spellberg B. The maturing antibiotic mantra: "shorter is still better". J Hosp Med 2018;13:361-2.

34. Mazzone A, Dentali F, La Regina M, et al. Clinical features, short-term mortality, and prognostic risk factors of septic patients admitted to Internal Medicine Units. Results of an Italian Multicenter Prospective Study. Medicine (Baltimore) 2016;95:e2124.

35. Virzì G, Day S, de Cal M, et al. Heart-kidney crosstalk and role of humoral signaling in critical illness. Crit Care 2014;18:201.

36. Spoto S, Valeriani E, Costantino S. Nosography of systemic inflammatory response syndrome, sepsis, severe sepsis, septic shock, and multiple organ dysfunction syndrome in internal medicine patients. Ital $\mathrm{J}$ Med 2015;9:243-51.

37. Spoto S, Navajas Martinez MF, Costantino S. Sepsis. Clin Ter 2002; 153:439-40.

38. Raj SK, Panda PK, Wig N, et al. Compliance with 6 hsepsis resuscitation bundle of surviving sepsis campaign before and after resident physicians' training: a quality improvement interventional study among Indian patients. J Emerg Trauma Shock 2019;12:3-9.2. 